\title{
Alternating Direction Method of Multipliers for Decentralized Electric Vehicle Charging Control
}

\author{
Jose Rivera, Philipp Wolfrum, Sandra Hirche, Christoph Goebel, and Hans-Arno Jacobsen
}

\begin{abstract}
The integration of Electric Vehicles (EVs) into the power grid is a challenging task. From the control perspective, one of the main challenges is the definition of a comprehensive control structure that is scalable to large EV numbers. This paper makes two key contributions: (i) It defines the EV ADMM framework for decentralized EV charging control. (ii) It evaluates EV ADMM using actual data and various EV fleet control problems. EV ADMM is a decentralized optimization algorithm based on the Alternating Direction Method of Multipliers (ADMM). It separates the centralized optimal fleet charging problem into individual optimization problems for the EVs plus one aggregator problem that optimizes fleet goals. Since the individual problems are coupled, they are solved consistently by passing incentive signals between them. The framework can be parameterized to trade-off the importance of fleet goals versus individual EV goals, such that aspects like battery lifetime can be considered. We show how EV ADMM can be applied to control an EV fleet to achieve goals such as demand valley filling and minimal-cost charging. Due to its flexibility and scalability, EV ADMM offers a practicable solution for optimal EV fleet control.
\end{abstract}

\section{INTRODUCTION}

Integrating large fleets of Electric Vehicles (EVs) into the power grid requires a comprehensive control structure. EVs represent a significant load increase to the system and without control they may cause stability problems and lead to an undesirable grid operation state [1]. However, EVs may also benefit the grid and improve its operation, e.g., by supplying regulation power [2]. If EVs are allowed to discharge, they could be used as mobile storage units. Such storage units would support the grid and help integrate renewable energy resources [3]. The challenge lies in the definition of a control structure that is able to deal with this large number of distributed devices.

The control of power system devices is usually done in a centralized manner, i.e., centralized optimization for openloop control. Many pilot projects on EV integration, like [4], follow this approach. However, the introduction of thousands or even millions of EVs as controllable devices would make a centralized optimization impracticable. The centralized optimization of as many as $50 \mathrm{EVs}$ can already be a daunting task (cf., [5]). To address this issue, we consider

\footnotetext{
Jose Rivera, Christoph Goebel and Hans-Arno Jacobsen are with the Department of Computer Science, Technische Universitat München, D-85748, Munich, Germany \{j.rivera, christoph.goebel\}atum.de, jacobsen@in.tum.de Philipp Wolfrum is with Siemens AG, Corporate Technology, D-81739, Munich, Germany philipp.wolfrumesiemens.com

Sandra Hirche is with the Institute for Information-oriented Control, Technische Universitat München, D-80290, Munich, Germany hirche@tum.de
}

a decentralized control structure, where computation is distributed across many nodes and coordinated by a central instance. Similar structures for decentralized EV charging control have already been applied to solve the valley filling problem in [6], [7] and [8]. However, many other relevant control objectives besides valley filling exist, such as price-based optimization [5], and direct coupling between Renewable Energy (RE) and EV demand [9]. It is not clear whether these control problems can also be solved using the methods proposed in [6], [7] and [8], since they require a strictly convex cost function. To address this shortcoming, in this paper we introduce EV ADMM, a decentralized optimization framework that is able to deal with various EV control problems. EV ADMM is based on the Alternating Direction Method of Multipliers (ADMM), which can deal with non-strictly convex cost functions. We build upon recent work in [10] that offers a framework for decentralized dynamic energy network management. Our contribution is to derive the ADMM algorithm focusing on EV charging and evaluate its performance by applying it to a range of EV charging control problems, including valley filling, price-based optimization, and direct coupling with renewable energy sources.

The remainder of this paper is organized as follows: In Section II, the optimal fleet charging problem and its solution via ADMM is presented. In Section III, we propose the EV ADMM framework and define its application to several EV control problems. In Section IV, we evaluate EV ADMM based on numerical simulations using actual data.

\section{Problem Formulation}

We consider a hierarchical control structure where an aggregator influences EV charging behavior through an incentive signal to reach certain goals. The aggregator could be any party interested in a particular aggregate EV fleet consumption profile, e.g., a utility or an Independent System Operator (ISO). As mentioned in [11] and [12], the goal of the aggregator is usually in conflict with the individual EV goals. While the aggregator wants to use EVs to minimize their operational costs, the individual EVs want to minimize their charging and battery depreciation costs. Any solution of the EV charging problem therefore has to trade-off the aggregator's goal against the goals of individual EVs. To address this, we define the optimal fleet charging problem as a joint optimization with a trade-off parameter $\gamma$ : 
TABLE I

OPTIMAL FLEET CHARGING PROBLEM VARIABLES DESCRIPTION

\begin{tabular}{|l|l|l|}
\hline Variable & Description & Type \\
\hline$x_{a}$ & Aggregated EV profile for all time slots & Vector $\in \mathbb{R}^{T}$ \\
$x_{i}$ & Profile of a single EV for all time slots & Vector $\in \mathbb{R}^{T}$ \\
$f_{a}\left(x_{a}\right)$ & Cost function of the aggregator & Convex function \\
$f_{i}\left(x_{i}\right)$ & Cost function of the EV $i$ & Convex function \\
$\mathbb{X}_{a}$ & Constraints set of the aggregator & Convex set \\
$\mathbb{X}_{i}$ & Constraints set of EV $i$ & Convex set \\
$\gamma$ & Goals trade-off parameter & Scalar \\
\hline
\end{tabular}

$$
\begin{array}{ll}
\underset{x_{a}, x_{i}}{\operatorname{minimize}} & f_{a}\left(x_{a}\right)+\gamma \sum_{i=1}^{N_{E V}} f_{i}\left(x_{i}\right) \\
\text { subject to } & x_{a}=\sum_{i=1}^{N_{E V}} x_{i} \\
& x_{a} \in \mathbb{X}_{a} \\
& x_{i} \in \mathbb{X}_{i} ; \quad i=1, \ldots, N_{E V} .
\end{array}
$$

The variables of the EV fleet charging problem are described in Table I. We use a discrete time model with time slots $t \in\{1, \ldots, T\}$. The aggregated EV charging profile is defined as the vector $x_{a}=\left[x_{a}(1), \ldots, x_{a}(T)\right]^{T}$. We consider a specific number of EVs defined by $N_{E V}$. The charging profile of each EV $i$ is defined by a vector $x_{i}=\left[x_{i}(1), \ldots, x_{i}(T)\right]^{T}$ for $i=1, \ldots, N_{E V}$. For an EV $i$ a value $x_{i}(t)>0$ means that the $\mathrm{EV}$ is consuming energy, i.e., charging, at time slot $t$. For $x_{i}(t)<0$ an EV $i$ is feeding energy back to the grid, i.e., discharging. The same convention is used for the aggregated EV profile $x_{a}$. Intuitively, the fleet charging optimization problem minimizes the aggregator and the EVs objective functions subject to its local individual constraints. However, the individual problems are coupled by the equilibrium constraint, $x_{a}=\sum_{i=1}^{N_{E V}} x_{i}$.

This problem can be formulated as a standard exchange optimization problem. To do this, we consider the EVs and the aggregator as agents and define the number of agents as:

$$
N=N_{E V}+1 .
$$

The aggregator is redefined as agent $N$, where:

$$
x_{N}=-x_{a} .
$$

The cost function of agent $N$ is:

$$
f_{N}\left(x_{N}\right)= \begin{cases}f_{a}\left(-x_{N}\right) & \text { if }-x_{N} \in \mathbb{X}_{a} \\ \infty & \text { otherwise. }\end{cases}
$$

The EVs are agents $i=1, \ldots, N-1$ and their cost function is:

$$
f_{i}\left(x_{i}\right)= \begin{cases}\gamma f_{i}\left(x_{i}\right) & \text { if } x_{i} \in \mathbb{X}_{i} \\ \infty & \text { otherwise }\end{cases}
$$

With this definition the optimal fleet charging problem becomes an exchange problem:

$$
\begin{array}{cl}
\underset{x_{i}}{\operatorname{minimize}} & \sum_{i=1}^{N} f_{i}\left(x_{i}\right) \\
\text { subject to } & \sum_{i=1}^{N} x_{i}=0 .
\end{array}
$$

The exchange problem considers $\mathrm{N}$ agents exchanging a common good under an equilibrium constraint. It is a canonical optimization problem with many applications. In economics, e.g., this formulation is used to solve market equilibrium problems. According to [10], the ADMM solution of the exchange problem is:

$$
\begin{aligned}
x_{i}^{k+1} & =\min _{x_{i}} f_{i}\left(x_{i}\right)+y^{k} x_{i}+\frac{\rho}{2}\left\|x_{i}-x_{i}^{k}+\bar{x}^{k}\right\|_{2}^{2} \\
y^{k+1} & =y^{k}+\rho \bar{x}^{k+1},
\end{aligned}
$$

where $k$ is the current iteration number, $\rho>0$ is the augmented Lagrangian parameter also known as penalty parameter, $\bar{x}=1 / N \sum_{i=1}^{N} x_{i}$ is the average profile of all the agents, and $y \in \mathbb{R}^{T}$ is the dual variable, which one can interpret as price vector. With $u=y / \rho$ the solution becomes:

$$
\begin{aligned}
x_{i}^{k+1} & =\min _{x_{i}} f_{i}\left(x_{i}\right)+\frac{\rho}{2}\left\|x_{i}-x_{i}^{k}+\bar{x}^{k}+u^{k}\right\|_{2}^{2} \\
u^{k+1} & =u^{k}+\bar{x}^{k+1}
\end{aligned}
$$

Using the exchange problem solution (8) and taking into account the definitions in (3), (4) and (5), we can formulate the ADMM solution to the optimal fleet charging problem as follows:

\section{EV ADMM}

1) For each $\mathrm{EV} i=1, \ldots, N_{E V}$ :

$$
\begin{array}{ll}
x_{i}^{k+1}= & \\
\underset{x_{i}}{\operatorname{minimize}} & \gamma f_{i}\left(x_{i}\right)+\frac{\rho}{2}\left\|x_{i}-x_{i}^{k}+\bar{x}^{k}+u^{k}\right\|_{2}^{2} \\
\text { subject to } & x_{i} \in \mathbb{X}_{i} .
\end{array}
$$

2) For the aggregator:

$$
\begin{array}{ll}
x_{N}^{k+1}= & \\
\underset{x_{N}}{\operatorname{minimize}} & f_{a}\left(-x_{N}\right)+\frac{\rho}{2}\left\|x_{N}-x_{N}^{k}+\bar{x}^{k}+u^{k}\right\|_{2}^{2} \\
\text { subject to } & -x_{N} \in \mathbb{X}_{a} .
\end{array}
$$

3) Incentive signal update:

$$
\begin{aligned}
& \bar{x}^{k+1}=\frac{1}{N} \sum_{i=1}^{N} x_{i}^{k+1} \\
& u^{k+1}=u^{k}+\bar{x}^{k+1} .
\end{aligned}
$$

The EVs and the aggregator solve the above optimization problems independently in each step $k$ while the continuous update of the incentive signal drives the solution to the optimum of the original EV fleet charging problem.

Convergence criteria: As defined in [13], the convergence criteria for ADMM are given by the primal feasibility $r^{k} \in$ $\mathbb{R}^{T}$ and the dual feasibility $s_{i}^{k} \in \mathbb{R}^{T}$ :

$$
\begin{aligned}
r^{k} & =\bar{x}^{k} \\
s_{i}^{k} & =-\rho N\left(x_{i}^{k}-x_{i}^{k-1}+\left(\bar{x}^{k-1}-\bar{x}^{k}\right)\right),
\end{aligned}
$$

with $s^{k}=\left[s_{1}^{k}, \ldots, r_{N}^{k}\right]^{T}$, the stopping criteria is:

$$
\begin{aligned}
& \left\|r^{k}\right\|_{2} \leq \varepsilon^{\text {pri }} \\
& \left\|s^{k}\right\|_{2} \leq \varepsilon^{\text {dual }},
\end{aligned}
$$

where $\varepsilon^{\text {pri }}>0$ and $\varepsilon^{\text {dual }}>0$. 
Penalty parameter $\rho$ : Although some guidelines for choosing $\rho$ can be found in [13] and [10], the literature does not offer a comprehensive method for this task. In this work we used empirical values.

\section{THE EV ADMM FRAMEWORK}

The EV ADMM framework is the application of the ADMM solution to the optimal fleet charging problem. Fig. 1 describes the framework using a sequence diagram. As we can see, the aggregator first sends an incentive signal to the EVs. This incentive signal is composed of the scaled price $u^{k}$ and the average profile of all agents $\bar{x}^{k}$. Based on this incentive signal, the EVs and the aggregator solve their individual optimization problem, defined in (9) and (10). The EVs then send their solution to the aggregator as an aggregated response, $\bar{x}_{E V}^{k+1}=\frac{1}{N} \sum_{k=1}^{N_{E V}} x_{i}^{k+1}$. This aggregated response can be computed by a router, who receives the individual EV responses and sends the aggregated response to the aggregator. The aggregator later adds its result to obtain the average of all agents, $\bar{x}^{k+1}=\bar{x}_{E V}^{k+1}+\frac{x_{N}^{k+1}}{N}$. With this value, the aggregator then updates the scaled price $u^{k+1}$ according to (12). This sequence is repeated until the convergence criteria in (15) are met.

Using EV ADMM, the aggregator and the EV optimization problems can be defined independently of each other. The solution to the original optimal fleet charging problem is obtained by incorporating these problems into the EV ADMM framework. Let us now define the EV ADMM formulation for some common aggregator and EV optimization problems. In the following, we consider convex optimization problems, which guarantee globally optimal solutions. Furthermore, to allow for a trade-off comparison between the aggregator and EV goals, the unit of their cost functions have to be equal, in this case they are measured in EUR.

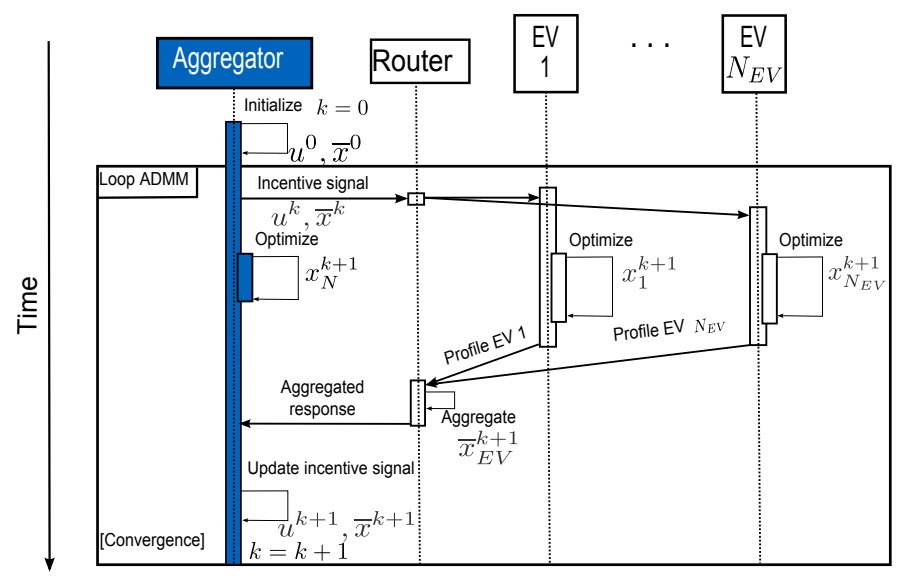

Fig. 1. EV ADMM sequence diagram.

\section{A. Aggregator optimization problem}

We defined three aggregator optimization problems that represent typical goals for EV fleet charging found in the literature. These lead to different objective functions $f_{a}\left(x_{a}\right)$ and constraint set $\mathbb{X}_{a}$ in problems (1) and (10), respectively.

Valley filling: In the valley filling problem, the goal is to flatten a given fixed demand profile as much as possible, e.g., by filling the overnight valley in the fixed demand with the demand caused by the EVs. Our formulation of the valley filling problem is similar to the one in [7]:

$$
\underset{x_{a}}{\operatorname{minimize}} \delta\left\|D+x_{a}\right\|_{2}^{2},
$$

where the fixed demand profile $D \in \mathbb{R}^{T}$ is assumed to be known and the parameter $\delta$ is empirically obtained by dividing the average energy price by the average of the fixed demand and multiplying it by the time step $\Delta t$. Parameter $\delta$ guarantees that the cost of the aggregator's optimization problem is in EUR.

Using the formulation in (10), the valley filling problem in the EV ADMM framework becomes:

$$
\min _{x_{N}}^{x_{N}^{k+1}}=\delta\left\|D-x_{N}\right\|_{2}^{2}+\frac{\rho}{2}\left\|x_{N}-x_{N}^{k}+\bar{x}^{k}-u^{k}\right\|_{2}^{2} .
$$

This problem can be solved analytically:

$$
\begin{aligned}
& x_{N}^{k+1}= \\
& \frac{\rho}{\rho-2 \delta}\left(x_{N}^{k}-\bar{x}^{k}+u^{k}\right)-\frac{2 \delta}{\rho-2 \delta} D .
\end{aligned}
$$

Price-based: In the case of a price-based optimization, the goal is to minimize the charging costs of an EV fleet while satisfying constraints on the minimal and maximal aggregated (dis)charging power. In [5], a centralized solution to this problem is presented, where the aggregated charging is constrained by the available renewable energy (RE) generation. Using EV ADMM, we can formulate a decentralized solution to this problem. We define the following aggregator problem:

$$
\begin{array}{ll}
\underset{x_{a}}{\operatorname{minimize}} & p^{T} x_{a} \Delta t \\
\text { s.t. } & \underline{x}_{a} \leq x_{a} \leq \bar{x}_{a},
\end{array}
$$

where $\Delta t$ is the time step duration. The maximal aggregated demand profile $\bar{x}_{a} \in \mathbb{R}^{T}$, the maximal power profile that can be fed back into the grid $\underline{x}_{a} \in \mathbb{R}^{T}$, and the electricity price $p \in \mathbb{R}^{T}$ are assumed to be known.

The price-based optimization problem in (19) is reformulated for the EV ADMM framework according to (10) as:

$$
\begin{array}{ll}
x_{N}^{k+1}= & \\
\min _{x_{N}} & -p^{T} x_{N} \Delta t+\frac{\rho}{2}\left\|x_{N}-x_{N}^{k}+\bar{x}^{k}+u^{k}\right\|_{2}^{2} \\
\text { s.t. } & -\underline{x}_{a} \geq x_{N} \geq-\bar{x}_{a} .
\end{array}
$$

Direct coupling: The direct coupling of RE and EVs considers that RE generation has been purchased to serve the EV demand. If the RE generation is not able to satisfy the EV demand, the required energy must be purchased on the spot market, which could be financially unattractive [9]. 
In our formulation RE generation excess is lost. Therefore, a careful matching should be made between RE production and EV fleet demand. The aggregator's role herein is to match EV demand and RE generation such that the cost of market purchased energy is minimized. For this case, we define the following optimization problem for the aggregator:

$$
\begin{array}{ll}
\underset{x_{p}, x_{a}}{\operatorname{minimize}} & p^{T} x_{p} \Delta t \\
\text { s.t. } & r e+x_{p} \geq x_{a},
\end{array}
$$

where $\Delta t$ is the time step duration and $x_{p} \in \mathbb{R}^{T}$ is the amount of extra power that needs to be purchased. The renewable energy power profile $r e \in \mathbb{R}^{T}$ and the energy price $p \in \mathbb{R}^{T}$ are known.

We introduce a slack variable $y_{\text {slack }} \in \mathbb{R}^{T}$ in order to transform the inequality constraint of our problem into an equality constraint. The problem then becomes:

$$
\begin{array}{ll}
\underset{x_{p}, x_{a}, y_{\text {slack }}}{\operatorname{minimize}} & p^{T} x_{p} \Delta t \\
\text { s.t. } & r e+x_{p}=x_{a}+y_{\text {slack }} \\
& y_{\text {slack }} \geq 0 .
\end{array}
$$

Considering that $x_{p}=y_{\text {slack }}-r e-x_{N}$ and after some mathematical reformulation, the direct coupling problem in (22) is reformulated using (10) for the EV ADMM framework. The resulting aggregator problem is then:

$$
\begin{array}{ll}
x_{N}^{k+1}= & \\
\min _{x_{N}, y_{\text {slack }}} & p^{T}\left(y_{\text {slack }}-x_{N}\right) \Delta t+\frac{\rho}{2}\left\|x_{N}-x_{N}^{k}+\bar{x}^{k}+u^{k}\right\|_{2}^{2} \\
\text { s.t. } & y_{\text {slack }} \geq 0 .
\end{array}
$$

\section{B. EV optimization problem}

For each EV $i$, we consider the following linear battery model:

$$
E_{i}(t+1)=E_{i}(t)+\Delta t x_{i}(t),
$$

where at time slot $t, E_{i}(t)$ is the energy stored in the battery and $x_{i}(t)$ is the power used to (dis)charge the battery. We assume that the EV's driving profile and energy requirements are known. We also assume as initial condition that the EV's battery is fully charged before its first trip. As in [5], we use this information and the battery model in (24) to formulate the charging requirements, as well as the bounds on the battery's energy state and power input, as series of inequality constraints that depend only on the EV's power profile $x_{i}$. With these constraints, we define the optimization problem of each $\mathrm{EV} i$ as:

$$
\begin{array}{ll}
\min _{x_{i}} & \alpha_{i}\left\|x_{i}\right\|_{2}^{2} \\
\mathrm{s.t} & \underline{R}_{i} \leq A_{i} x_{i} \leq \bar{R}_{i} \\
& \underline{S}_{i} \leq B_{i} x_{i} \leq \bar{S}_{i} \\
& \underline{x}_{i} \leq x_{i} \leq \bar{x}_{i} .
\end{array}
$$

The variables description of problem (25) can be found in Table II, where $c_{i}$ is the number of times that the EV is connected to the grid and $T_{c_{i}}$ is the total number of time slots that the EV is connected during the entire optimization horizon. We model the EV's goal of minimizing its battery
TABLE II

EV OPTIMIZATION PROBLEM VARIABLES DESCRIPTION

\begin{tabular}{|l|l|l|}
\hline Variable & Description & Type \\
\hline$x_{i}$ & EV charging profile & Vector $\in \mathbb{R}^{T}$ \\
$\underline{x}_{i}$ & Minimal charging power & Vector $\in \mathbb{R}^{T}$ \\
$\bar{x}_{i}$ & Maximal charging power & Vector $\in \mathbb{R}^{T}$ \\
$\alpha_{i}$ & Battery depreciation parameter & Scalar \\
$A_{i}$ & Connection matrix & Matrix $\in \mathbb{R}^{c_{i} \times T}$ \\
$B_{i}$ & Input matrix & Matrix $\in \mathbb{R}^{T_{c_{i}} \times T}$ \\
$\underline{R}_{i}$ & Minimal charging requirements & Vector $\in \mathbb{R}^{c_{i}}$ \\
$\bar{R}_{i}$ & Maximal charging requirements & Vector $\in \mathbb{R}^{c_{i}}$ \\
$\underline{S}_{i}$ & Minimal state charging power & Vector $\in \mathbb{R}^{T_{c_{i}}}$ \\
$\bar{S}_{i}$ & Maximal state charging power & Vector $\in \mathbb{R}^{T_{c_{i}}}$ \\
\hline
\end{tabular}

depreciation costs as a quadratic cost function of charging power. This cost function corresponds to the objective function $f_{i}\left(x_{i}\right)$ in problems (1) and (9). The first set of inequality constraints, $\underline{R}_{i} \leq A_{i} x_{i} \leq \bar{R}_{i}$, defines a bound on the energy requirements of the $\mathrm{EV}$. Each row represents an energy requirement for each time the EV is connected. The second set of inequality constraints, $\underline{S}_{i} \leq B_{i} x_{i} \leq \bar{S}_{i}$, guarantees, that the power input does not violate the maximal and minimal energy that the battery can support at any time slot when the EV is connected. These constraints contain the EV dynamics while connected. Thus, matrix $B_{i}$ is actually the input matrix that results from Equation (24) for the time slots when the EV is connected. The last set of constraints, $\underline{x}_{i} \leq x_{i} \leq \bar{x}_{i}$, define the maximal and minimal EV (dis)charging power for the optimization horizon. If $\mathrm{EV} i$ is not connected at time $t$, then $\underline{x}_{i}(t)=\bar{x}_{i}(t)=0$, else $\underline{x}_{i}(t)=x_{i}^{\text {min }}$ and $\bar{x}_{i}(t)=x_{i}^{\max }$, where $x_{i}^{\text {min }}$ and $x_{i}^{\max }$ are the maximal and minimal power that the EV charger supports. For more details on how to derive these parameters we refer the reader to [14].

For EV ADMM, the EV optimization problem in (25) is reformulated according to (9) into the following problem:

$$
\begin{array}{ll}
x_{i}^{k+1}= & \\
\min _{x_{i}} & \gamma \alpha_{i}\left\|x_{i}\right\|_{2}^{2}+\frac{\rho}{2}\left\|x_{i}-x_{i}^{k}+\bar{x}^{k}+u^{k}\right\|_{2}^{2} \\
\operatorname{s.t} & \underline{R}_{i} \leq A_{i} x_{i} \leq \bar{R}_{i} \\
& \underline{S}_{i} \leq B_{i} x_{i} \leq \bar{S}_{i} \\
& \underline{x}_{i} \leq x_{i} \leq \bar{x}_{i} .
\end{array}
$$

In this optimization problem, the scaled price $u$ can be considered an energy price that the aggregator defines for the EVs. The mean value of all agents $\bar{x}$ can be thought of as a social cost caused by the EVs not cooperating to achieve global convergence. Upon convergence this social cost should be close to zero.

\section{Evaluation}

In this Section, we evaluate the three aggregator control problems formally defined in Section III: Valley filling, price-based optimization, and direct coupling with renewables.

For each of these problems, we simulated EVs charging 
TABLE III

EVALUATION PARAMETERS

\begin{tabular}{|l|l|l|}
\hline Variable & Description & Value \\
\hline \multicolumn{3}{|c|}{ Electric Vehicle parameters } \\
\hline Cap ${ }_{\text {nom }}$ & Nominal battery capacity & $20 \mathrm{kWh}$ \\
$E_{\text {bat }}$ & Effective energy capacity & $0.85 * 20 \mathrm{kWh}$ \\
Con & Power Consumption & $0.15 \mathrm{kWh} / \mathrm{km}$ \\
$\alpha_{i}$ & Battery depreciation parameter & $0.0125 \mathrm{EUR} / \mathrm{kW}^{2}$ \\
$x_{i}^{\text {max }}$ & Maximal charging power & $4 \mathrm{~kW}$ \\
$x_{i}^{\text {min }}$ & Maximal discharging power & $-4 \mathrm{~kW}$ \\
\hline \multicolumn{3}{|c|}{ Valley filling parameters } \\
\hline$\delta$ & Empirical parameter \\
$\varepsilon^{\text {pri }}$ & Primal feasibility threshold & $2.95 * 10^{-5} \frac{\mathrm{EUR}}{\mathrm{kW}^{2}}$ \\
$\varepsilon^{\text {dual }}$ & Dual feasibility threshold & 20 \\
\hline \multicolumn{3}{|c|}{ Price-based parameters } \\
\hline$x_{a}$ & Aggregated maximal energy feed back & $-137.6 \mathrm{~kW}$ \\
$\bar{x}_{a}$ & Aggregated maximal consumption & $137.6 \mathrm{~kW}$ \\
$\varepsilon^{\text {pri }}$ & Primal feasibility threshold & 0.1 \\
$\varepsilon^{\text {dual }}$ & Dual feasibility threshold \\
\hline \multicolumn{3}{|c|}{ Direct coupling parameters } \\
\hline$\varepsilon^{\text {pri }}$ & Primal feasibility threshold \\
$\varepsilon^{\text {dual }}$ & Dual feasibility threshold & 0.1 \\
\hline
\end{tabular}

minimal energy, i.e., EVs only charge the amount of energy needed for their trips. We therefore assume that EVs are able to accurately predict their driving energy requirements for each trip. The option of having Vehicle-to-Grid (V2G) services, i.e., allowing the EVs to feed energy back to the grid, and different trade-off parameters $\gamma$ for the individual EV goals were also considered. We assume a time horizon of 24 hours and a 15 minute time step duration.

Our EVs are based on the MovE specifications [15], an EV developed by Siemens. We obtained vehicle trip patterns from the National Household Travel Survey (NHTS) data using the method described in [2]. The NHTS data set contains trips reported by more than 150,000 U.S. households [16]. Based on this data we computed the EVs charging requirements for each connection period. The fixed demand and renewable in-feed profiles, $D$ and $r e$, were obtained from the Munich distribution system operator website [17]. The latter profiles were scaled to correspond with the power consumption of 100 EVs. Finally, the energy price profile $p$ was obtained from the European Energy Exchange (EEX) website [18]. All the mentioned data is publicly available and corresponds to Nov 21, 2011. Other relevant parameters for our evaluation can be found in Table III. We simulated a sequential implementation of EV ADMM, i.e., the agents solve their optimization problems one at a time. One ADMM iteration is finished once all agents have found the optimal solution of their subproblem in that iteration. The simulation environment was MATLAB and for the optimizations we used CVXGEN [19]. Table IV provides an overview of the results obtained.


Fig. 2. Valley filling for $100 \mathrm{EVs}$ charging minimal energy. Left without V2G and right with V2G for different individual EV goal importance, $\gamma=$ $0,0.01,1$.

Valley filling: The goal in valley filling is to use the EVs to fill the overnight valley in the fixed demand. As seen in Fig. 2, valley filling is achieved if the individual EV goals are ignored, i.e., $\gamma=0$. Moreover, allowing V2G services results in an almost flat aggregated demand profile. However, our evaluation shows that, allowing V2G services does not translate into a major cost reduction. In Table IV, we can see that the cost difference between V2G and no V2G for the same $\gamma$ value is very small. Nevertheless, an all flat aggregated demand profile offers several advantages in terms of grid operation that are not taken into account by our cost function, e.g., the fact that with a flat valley no expensive peak plants need to be turned on.

Price-based: Here, the goal is to minimize the charging cost of an EV fleet and maintain the fleet demand/energy feedback within a given bound. To achieve this, the aggregator modifies the energy price it gives to the EVs. Fig. 3 shows the modified energy price and the fleet behavior for the V2G case. We can see that the price only changes if the EVs meet the bound. We can also see in Table IV, that for low $\gamma$ values allowing V2G services significantly reduces the costs for the whole system.

TABLE IV

PERFORMANCE METRICS FOR DIFFERENT IMPORTANCE LEVELS OF INDIVIDUAL EV GOALS $\gamma$

\begin{tabular}{|l|l|l|l|}
\hline & Number of ADMM iterations - Total cost [EUR] \\
\hline & $\gamma=0$ & $\gamma=0.01$ & $\gamma=1$ \\
\hline \multicolumn{3}{|c|}{ Valley filling } \\
\hline No V2G & $293-693$ & $283-693$ & $275-737$ \\
V2G & $293-687$ & $293-689$ & $290-737$ \\
\hline \multicolumn{3}{|c|}{ Price-based } \\
\hline No V2G & $824-41$ & $881-42$ & $664-79$ \\
V2G & $1394-27$ & $1266-31$ & $664-79$ \\
\hline \multicolumn{3}{|c|}{ Direct coupling } \\
\hline No V2G & $341-13$ & $284-12$ & $328-53$ \\
V2G & $485-12$ & $300-13$ & $278-53$ \\
\hline
\end{tabular}




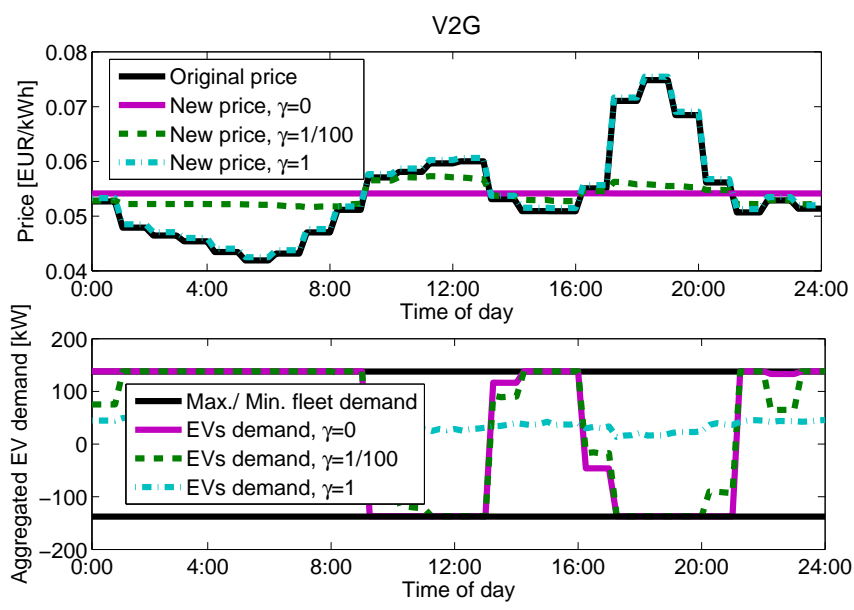

Fig. 3. Price-based optimization for 100 EVs charging minimal energy with V2G. Above the optimal incentive price and bellow the aggregated fleet demand, both for different individual EV goal importance values, $\gamma=$ $0,0.01,1$.

Direct coupling: In direct coupling the aggregator has purchased renewable energy to supply the EVs' demand. As in the price-based problem, the aggregator modifies the price as an incentive for the EVs to absorb this renewable energy. This lowers the aggregator's energy sourcing costs. Table IV reveals that even with low $\gamma$ values, V2G services do not produce any significant cost reduction. Therefore, based on our evaluation, V2G services do not offer a major cost advantage in the direct coupling of RE generation and EVs consumption.

\section{COnClusions}

In this work, we introduced EV ADMM as a framework for decentralized EV charging control. We provided the implementation details for several EV fleet control problems: Valley filling, price-based optimization and direct coupling of EVs and renewable energy. The evaluation shows that, for $100 \mathrm{EVs}$, a sequential EV ADMM is able to deliver results in an average time of 2 minutes. As discussed in [10], ADMM has a linear computational complexity with respect to the number of agents. Therefore, we can estimate that EV ADMM has a linear computational complexity with respect to the number of EVs. In a parallel implementation, the decentralized structure of EV ADMM can offer great scalability advantages over a centralized implementation. Our simulation-based evaluation also showed that the relative weight of the individual $\mathrm{EV}$ goals has to be rather small to make the control approach useful on the system level.

We have shown that EV ADMM can be implemented in a decentralized manner for various EV fleet control problems. However, the communication overhead resulting from the ADMM algorithm makes it only practical for offline calculations. Nevertheless, the flexibility and scalability that EV ADMM offers are a necessary requirement for the optimal control of large EV fleets.

\section{REFERENCES}

[1] M. Singh, I. Kar, and P. Kumar, "Influence of EV on grid power quality and optimizing the charging schedule to mitigate voltage imbalance and reduce power loss," in Power Electronics and Motion Control Conference (EPE/PEMC), 2010 14th International, sept. 2010, pp. T2-196-T2-203.

[2] C. Goebel and D. S. Callaway, "Using ICT-Controlled Plug-in Electric Vehicles to Supply Grid Regulation in California at Different Renewable Integration Levels," Smart Grid, IEEE Transactions on, vol. PP, no. 99 , pp. 1-12, 2012.

[3] S. Babaei, D. Steen, L. A. Tuan, O. Carlson, and L. Bertling, "Effects of Plug-in Electric Vehicles on distribution systems: A real case of Gothenburg," in Innovative Smart Grid Technologies Conference Europe (ISGT Europe), 2010 IEEE PES, oct. 2010, pp. 1-8.

[4] C. Binding, D. Gantenbein, B. Jansen, O. Sundstrom, P. Andersen, F. Marra, B. Poulsen, and C. Traeholt, "Electric vehicle fleet integration in the danish EDISON project - A virtual power plant on the island of Bornholm," in Power and Energy Society General Meeting, 2010 IEEE, july 2010, pp. 1-8.

[5] O. Sundström and C. Binding, "Optimization Methods to Plan the Charging of Electric Vehicle Fleets," in Proc. Int'l Conf. on Control, Communication and Power Engineering (CCPE), San Diego, CA, 2010, pp. 323-328.

[6] Z. Ma, D. Callaway, and I. Hiskens, "Decentralized charging control for large populations of plug-in electric vehicles: Application of the Nash certainty equivalence principle," in Control Applications (CCA), 2010 IEEE International Conference on, sept. 2010, pp. 191-195.

[7] L. Gan, U. Topcu, and S. Low, "Optimal decentralized protocol for electric vehicle charging," in Decision and Control and European Control Conference (CDC-ECC), 2011 50th IEEE Conference on, dec. 2011, pp. 5798-5804.

[8] L. Gan, U. Topcu, and S. H. Low, "Stochastic decentralized protocols for electric vehicle charging with discrete charging rate," in IEEE Power and Energy Society General Meeting, San Diego, CA, July 2012

[9] A. Papavasiliou, S. Oren, and R. O'Neill, "Reserve Requirements for Wind Power Integration: A Scenario-Based Stochastic Programming Framework," Power Systems, IEEE Transactions on, vol. 26, no. 4, pp. 2197-2206, nov. 2011.

[10] M. Kraning, E. Chu, J. Lavaei, and S. Boyd, "Message Passing for Dynamic Network Energy Management," To Appear: Foundations and Trends in Optimization, Apr. 2012.

[11] N. Leemput, J. Van Roy, F. Geth, P. Tant, B. Claessens, and J. Driesen, "Comparative analysis of coordination strategies for electric vehicles," in Innovative Smart Grid Technologies (ISGT Europe), 2011 2nd IEEE PES International Conference and Exhibition on, dec. 2011, pp. 1-8.

[12] D. Callaway and I. Hiskens, "Achieving Controllability of Electric Loads," Proceedings of the IEEE, vol. 99, no. 1, pp. 184-199, jan. 2011

[13] S. Boyd, N. Parikh, E. Chu, B. Peleato, and J. Eckstein, "Distributed Optimization and Statistical Learning via the Alternating Direction Method of Multipliers," Foundation and Trends in Machine Learning, vol. 3, no. 1, pp. 1-122, 2011.

[14] J. Rivera, "Optimal Charging of Electric Vehicles with Renewable Energy in Smart Grids," Master thesis, Technische Universität München, Nov 2012.

[15] SIEMENS, “Just Plug 'er in!” Apr. 2011.

[16] C. Goebel, "On the business value of ICT-controlled plug-in electric vehicle charging in California," Energy Policy, August 2012.

[17] SWM München, “Netzdaten," 2012. [Online]. Available: http://www.swm-infrastruktur.de/strom/netzstrukturdaten/netzdaten.html

[18] E. E. Exchange, "European Electricity Index," http://www.eex.com/en/, 2012.

[19] J. Mattingley and S. Boyd, "CVXGEN: A Code Generator for Embedded Convex Optimization," Optimization and Engineering, vol. 13, no. 1, pp. 1-27, March 2012. 